\title{
Remoción de semillas por roedores en un fragmento de bosque seco tropical (Risaralda-Colombia)
}

\author{
Seed removal by rodents in a fragment of dry tropical forest \\ (Risaralda-Colombia)
}

\author{
Felipe Vélez-García, ${ }^{1 *}$ B.Sc, Jairo Pérez-Torres, ${ }^{1}$ Ph.D. \\ ${ }^{1}$ Pontificia Universidad Javeriana, Unidad de Ecología y Sistemática (UNESIS), Laboratorio de \\ Ecología Funcional (LEF). Cra. 7 No. 43-82, Edificio 54, Laboratorio 406B. Bogotá D.C., Colombia. \\ ${ }^{*}$ Correspondencia:velez.j@javeriana.edu.co
}

Recibido: Octubre 14 de 2009; Aceptado: Marzo 21 de 2010.

\section{RESUMEN}

Objetivos. Los roedores son los mayores depredadores de semillas en ecosistemas neotropicales, sin embargo, la fragmentación afecta su presencia y por ende la depredación de semillas. Materiales y métodos. Se reconoció el porcentaje y la tasa de remoción de semillas por roedores en zonas de interior, borde y pastizal de un fragmento de bosque seco en el sector Cerritos - La Virginia (Risaralda-Colombia). Entre marzo y julio de 2003 se identificaron los roedores presentes en el bosque con la ayuda de 60 trampas Sherman ubicadas en diferentes zonas del fragmento. Para obtener el porcentaje y la tasa de remoción de semillas por roedores fueron aplicados dos experimentos (primero en junio y el segundo en julio) con un diseño de bloques aleatorios usando tres tipos de encierros: total (acceso a insectos), parcial (acceso a roedores) y control (acceso a cualquier organismo), teniendo en cuenta la ubicación en el fragmento (interior-borde-pastizal). Durante el primer experimento (junio) fueron utilizadas7200 semillas de Samanea saman y 6000 semillas durante el segundo (julio). Resultados. Se capturaron 4 individuos de Heteromys australis al interior del bosque. En junio 1577 (44.87\%) semillas fueron removidas por los roedores al interior del bosque. En julio se removieron 1620 semillas de las cuales el 60.5\% fue por roedores al interior del bosque. Conclusiones. Los resultados reflejan una mayor remoción de semillas por roedores al interior del bosque donde el riesgo de depredación y la disponibilidad de refugio son más altos.

Palabras clave: Depredación, semillas, Samanea saman, roedores, Heteromys australis, Colombia. 


\section{ABSTRACT}

Objectives. Rodents are the biggest seed predators in Neotropical ecosystems, however, fragmentation affects their presence, therefore seed predation. Materials and methods. The percentage and removal rate of seeds by rodents was recognized in areas of interior, borderline and pasture of a dry forest in the area of Cerritos - La Virginia (RisaraldaColombia). Between March and July 2003, rodents present in the forest were identified with the aid of 60 Sherman traps located in different areas of the fragment. To obtain the percentage and removal rate of seeds by rodents, two experiments were conducted, (the first in June and the second one in July) with a random block design, using three types of closures: total (access to insects), partial (access to rodents) and control, (access to any organism), taking into account the location within the fragment (interior, borderline, or pasture). During the first experiment (June) 2700 seeds of Samanea saman were used and during the second one (July) 6000 seeds were used. Results. Four individuals of the Heteromys australis species were captured in the interior of the forest. In June 1577 $(44.87 \%)$ seeds were removed out by rodents within the interior of the forest. In July 1620 $(60.5 \%)$ seeds were removed out by rodents within the interior of the forest. Conclusions. The results show a higher seed removal by rodents within the interior of the forest where the risk of predation and refuge availability is higher.

Key words: Predation, seeds, Samanea saman, rodents, Heteromys australis, Colombia.

\section{INTRODUCCIÓN}

La depredación de semillas juega un papel importante en la dinámica poblacional de las plantas, limitando la regeneración natural de los bosques y afectando su composición y estructura (1). Los roedores son de gran importancia en la ecología de las plantas gracias a procesos como la dispersión de semillas $(2,3)$, el establecimiento de plántulas $(1,4-6)$ y la depredación de semillas $(1,3,6-9)$, siendo organismos modelo para identificar los efectos ocasionados por esta en la distribución y dinámica de poblaciones vegetales (8).

En ecosistemas neotropicales los roedores son considerados los mayores consumidores de semillas (1,10-12). Sin embargo, problemas como la fragmentación alteran la presencia de roedores en un área determinada, afectando también procesos como la remoción y depredación de semillas y por consiguiente la regeneración de bosques y de áreas degradadas $(7,11,13)$.

El uso del espacio por roedores para el forrajeo varía en función de factores como: la cantidad de luz, parámetros meteorológicos asociados, la densidad y tipo de cobertura vegetal a nivel del suelo y del dosel, la competencia por explotación de recursos y la distancia a diferentes límites (bordes) de vegetación $(4,14-16)$. Estos factores se encuentran asociados a la fragmentación de los bosques y a condiciones resultantes de la fragmentación como el tamaño y la forma del fragmento y el tipo de matriz circundante (17). En este sentido la fragmentación de los bosques afecta la presencia y actividad de los roedores en los ecosistemas, especialmente en hábitats de borde donde la densidad y actividad varían dependiendo de la capacidad de adaptación de las especies a las nuevas condiciones resultantes de la fragmentación $(4,14,18)$. Dado lo anterior la depredación de semillas en hábitats fragmentados y especialmente en los bordes puede variar dependiendo de la disponibilidad de semillas y de la estructura y composición del ensamblaje de roedores, presentando un efecto cascada en la interacción planta-roedor $(11,19)$.

Aunque se han desarrollado diversos estudios para evaluar el efecto de borde sobre la remoción o depredación de semillas, los resultados son contradictorios respecto al hábitat (interior o borde) en el que se presenta mayor depredación $(11,19)$. La 
depredación de semillas y el reclutamiento de plántulas es un proceso complejo de múltiples estados y aún es poca la información generada para entender bien este proceso especialmente en su relación con el efecto de borde en ambientes fragmentados (19).

En Colombia, los bosques secos del Valle del Cauca y los sub-húmedos de Risaralda son de gran importancia para el sostenimiento de la fauna, así como para la regulación hídrica de la zona (20). Actualmente la deforestación a causa de la agricultura, la ganadería extensiva y el urbanismo son las principales amenazas que enfrenta (20).

El objetivo de este trabajo fue reconocer si el porcentaje y la tasa de remoción de semillas por roedores cambian de acuerdo con el tipo de hábitat (interior-borde-exterior) en un fragmento de bosque montano seco en el sector Cerritos-La Virginia, municipio de Pereira (Risaralda- Colombia).

\section{MATERIALES Y MÉTODOS}

Área de estudio. El estudio se desarrolló en la Hacienda Cristales, Vereda el Cauquillo, sector Cerritos-La Virginia, municipio de Pereira (Risaralda), Colombia. Esta comprende una zona boscosa transicional entre bosque seco tropical (bs-T) y bosque húmedo premontano (bh-PM), alrededor de los ríos Otún y Cauca, ubicada entre 900 y $1200 \mathrm{~m}$ de altitud, con una extensión aproximada de 96.25 ha. Presenta una precipitación anual de 1500-3000 mm/ha, con una humedad relativa de $70-80 \%$ y una temperatura promedio anual de $18-24^{\circ} \mathrm{C}$ (20).

La vegetación más común está compuesta por matorrales donde domina la zarza (Mimosa pigra), guayabo de loma (Psodium guineensis), maugle (Escallonia sp.) y el rascador (Mauria ovalifolia). En las vegas de los ríos se encuentra cañabrava (Gynerium sagittarium), carbonero (Calliandra sp.), chagualo (Rapanea guianensis), cedrillo (Guarea sp.), moro (Brysonima cumingana), cedro (Cederia sp.), cassias y cámbulos. Actualmente también se aprecian potreros de ganadería extensiva y pequeños relictos de Guadua (Guadua angustifolia) (20).

Métodos. El estudio se desarrolló de marzo a julio de 2003 (correpondiente a los periodos lluvioso y seco en el área de estudio) teniendo en cuenta dos componentes: 1) captura de roedores y 2) experimentos de remoción de semillas.

Captura de roedores. Para la captura de roedores se utilizaron 60 trampas Sherman $8 \mathrm{~cm} \times 9 \mathrm{~cm} \times 23 \mathrm{~cm}$, en las que se utilizó como cebo avena en hojuelas y mantequilla de maní. Entre marzo y junio de 2003 las trampas fueron instaladas en diferentes puntos del pastizal y del bosque, buscando los sitios más propicios para la presencia de roedores. Las trampas se mantuvieron activas las 24 horas del día, para un esfuerzo de muestreo de 2552 trampas-noche al interior del bosque y 480 trampas-noche en el pastizal. En junio, tiempo en el que se iniciaron los experimentos de remoción de semillas, las trampas fueron colocadas entre los transectos experimentales (al interior y en el borde) a excepción del pastizal, ya que no hubo indicios de roedores durante los muestreos anteriores en esta zona. En cada una de las zonas se trazó un transecto de $130 \mathrm{~m}$, separado a un mínimo de $35 \mathrm{~m}$ de los transectos del experimento. En cada transecto se colocaron 14 trampas Sherman separadas cada 10 metros. Los ejemplares capturados fueron medidos y pesados para su posterior identificación.

Remoción de semillas. Para medir la remoción de semillas se realizaron dos experimentos de exclusión (en los meses de junio y julio, respectivamente) utilizando hábitat de interior, borde y pastizal. En cada hábitat se trazaron dos transectos de $180 \mathrm{~m}$ cada uno, manteniendo una distancia mínima de 200 m entre el final del primer transecto y el comienzo del segundo, y ubicados de forma paralela al borde del bosque. Los transectos del interior fueron trazados en el centro del fragmento, los del borde fueron trazados a una distancia de $10 \mathrm{~m}$ desde el borde físico hacia el interior del fragmento de bosque y los transectos del pastizal fueron trazados a $10 \mathrm{~m}$ contados desde el borde físico del bosque hacia el exterior. 
En cada transecto, se ubicaron 10 parcelas experimentales de $2 \times 2 \mathrm{~m}$, distanciadas 20 $\mathrm{m}$ desde el centro de una parcela hasta el centro de la siguiente. Cada parcela fue divida en cuatro cuadrantes de $1 \times 1 \mathrm{~m}$, de los cuales se escogieron aleatoriamente tres para ubicar los tratamientos.

Se establecieron tres tratamientos: 1) Tratamiento "exclusión total", el cual permitió la entrada de invertebrados pero evitó el ingreso de roedores, aves y otros vertebrados predadores de semillas. 2) Tratamiento "exclusión parcial", que permitió el ingreso de insectos y roedores, pero impidió el acceso de otros organismos como aves y mamíferos medianos y grandes. 3) Tratamiento "sin exclusión" el cual permitió el acceso a cualquier organismo presente en la zona.

Los tratamientos consistieron en colocar, a manera de encierro, cubos de malla plástica de $20 \times 20 \times 10 \mathrm{~cm}$, con un ojo de $0.5 \times 0.5 \mathrm{~cm}$, en el centro de cada uno de los cuadrantes previamente seleccionados. Para el tratamiento "exclusión total" (ET), las caras laterales de los cubos de malla se dejaron intactos (cerrados), mientras que en el tratamiento "exclusión parcial" (EP), se hicieron orificios de $5.0 \mathrm{~cm}$ de ancho $x$ $3.5 \mathrm{~cm}$ de alto en las caras laterales del cubo, para permitir el ingreso de roedores, manteniendo los demás lados cerrados. Para el tratamiento "sin exclusión" (SE) no se utilizó encierro, permitiendo el acceso a las semillas por parte de cualquier organismo del bosque. En cada encierro se colocó un plato de icopor de $12 \mathrm{~cm}$ de diámetro, sobre el cual se depositaron 40 semillas de Samanea saman, la cual es una especie característica de bosque seco tropical, presente en pastizales y zonas abiertas, y con alto potencial agroforestal (21). Las semillas cuyo tamaño promedio es de 1.0 $\mathrm{cm}$ de largo por $0.7 \mathrm{~cm}$ de diámetro fueron colectadas de diferentes árboles presentes en la zona.

El primer experimento se llevó a cabo del 9 al 20 de junio de 2003. Para este experimento se utilizó un total de 7200 semillas las cuales se revisaron cada tercer día. Se registró el número de semillas removidas o con marcas de depredación (roídas o con infección de escarabajos) que implicaran que ya no fuesen viables. El tiempo de duración del experimento se determinó como el momento en que se removió el 50\% (800 semillas) de las semillas de alguno de los tratamiento con acceso a roedores (tratamientos EP y $\mathrm{SE}$ ) en cualquiera de los hábitats (interior, borde y pastizal). Para el caso del pastizal, solo se obtuvieron datos de remoción de uno de los transectos trazados ya que el ganado presente en los pastizales destruyó las parcelas experimentales.

El segundo experimento se desarrolló del 13 al 25 de julio de 2003. Para este se utilizaron 6000 semillas de S. saman debido a la poca disponibilidad de semillas durante esta época del año; en este caso solo se utilizó un transecto en el borde, mientras que para el interior y el pastizal se utilizaron los mismos transectos del primer experimento. La revisión se realizó cada tres días registrando el número de semillas removidas o con marcas de depredación. El tiempo de finalización para este experimento fue el mismo que en el primer experimento.

Análisis de información. Para analizar la remoción de semillas se calculó un valor de remoción neta, que permitió una aproximación a la posible remoción por roedores (para el caso del tratamiento EP) y otros vertebrados de mediano o gran tamaño (tratamiento SE).

La remoción neta se calculó restando los valores de remoción total (observada en campo) para los tratamientos de la siguiente manera: Para el tratamiento EP (remoción por roedores), se restaron los valores de la remoción observada en el tratamiento ET (remoción por insectos), para el tratamiento SE (otros vertebrados) se restaron los valores de remoción observada en los tratamientos ET (remoción por insectos) y EP (remoción por roedores). Los valores de remoción neta obtenidos para los tratamientos, fueron los valores utilizados para obtener el porcentaje de la remoción de semillas por roedores en cada hábitat en los que se desarrollaron los experimentos. La remoción neta se analizó mediante la prueba Kruskal-Wallis teniendo en cuenta dos comparaciones: 1) entre 
tratamientos (ET, EP y SE), 2) respecto a la ubicación (interior, borde y pastizal). Las tasas de remoción se compararon mediante la prueba G.

\section{RESULTADOS}

Captura de roedores. Se capturaron cuatro individuos (tres hembras y un macho) de Heteromys australis (Heteromyidae), al interior del bosque entre marzo y junio; el éxito de captura fue de 0.0015 individuos por 100 trampas-noche.

Remoción de semillas. Para el primer experimento (junio) se removieron 1577 semillas luego de 12 días de iniciado. Durante este tiempo se consumieron más del $50 \%$ (852) de las semillas con acceso a roedores, al interior del bosque.

Para el porcentaje de remoción de semillas se encontraron variaciones entre tratamientos al interior del fragmento $(H=26.13, n=55$, $\mathrm{gl}=2, \mathrm{p}<0.05$ ) (Figura 1a) y en el borde $(H=32.01, n=56, g l=2, p<0.05)$ (Figura 1b), mientras que en el pastizal las diferencias no fueron significativas $(H=4.31, n=26$,
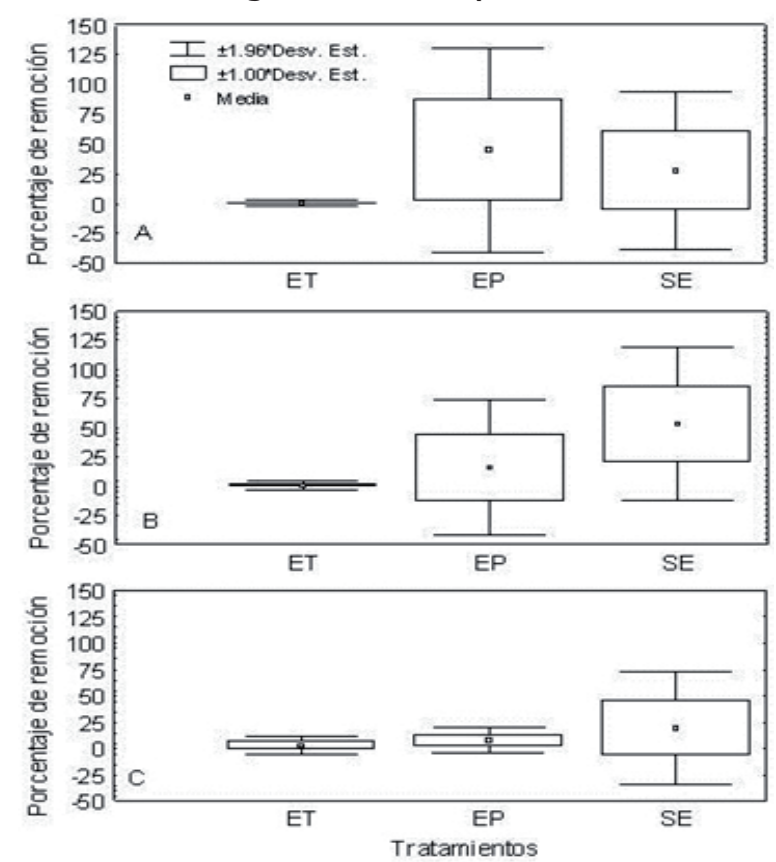

Figura 1. Comparación entre el porcentaje de remoción de semillas entre tratamientos del experimento 1 (junio). A) Interior. B) Borde C) Pastizal. $\mathrm{ET}=$ Exclusión total. $\mathrm{EP}=$ Exclusión parcial, $\mathrm{SE}=$ Sin exclusión. $\mathrm{gl}=2, \mathrm{p}>0.05$ ) (Figura 1c); en este caso las mayores diferencias se presentaron para tratamiento EP al interior y para el tratamiento SE en el borde y el pastizal.

En cuanto a la ubicación (comparación entre hábitats para un mismo tratamiento) se encontró mayor remoción al interior del fragmento (855 semillas), y menor en pastizal (134 semillas). El tratamiento ET presentó mayor porcentaje de remoción de semillas en el pastizal $(3 \%)$ y menor el interior $(0.38 \%)(H=4.31, n=50, g l=2$, $\mathrm{p}>0.05$ ) (Figura 2a). El tratamiento EP presentó el mayor porcentaje de remoción al interior (44.87\%) y el menor en el pastizal $(4.25 \%)$, sin embargo las diferencias no fueron significativas $(H=5.88, n=47$, $\mathrm{gl}=2, \mathrm{p}>0.05$ ) (Figura 2b). Finalmente el
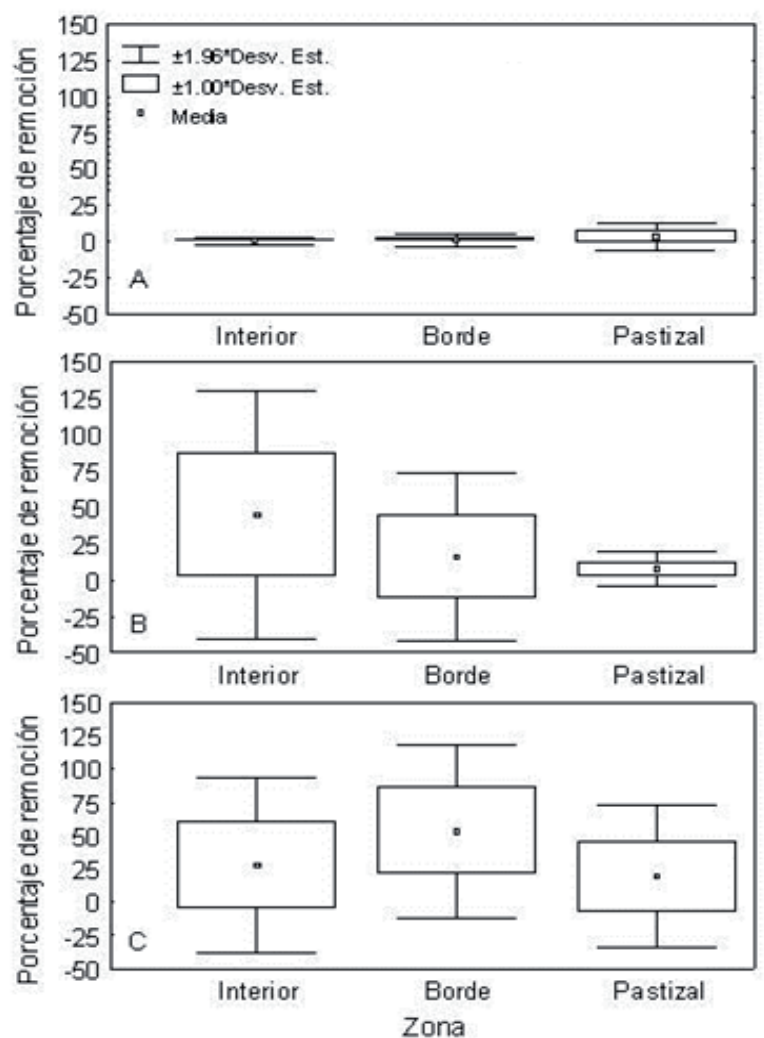

Figura 2. Comparación entre el porcentaje de remoción y la zona trabajada en el fragmento de bosque para cada tratamiento del experimento 1 (junio). A). Exclusión Total. B). Exclusión Parcial. C). Sin Exclusión.

tratamiento se presentó un porcentaje de remoción significativamente mayor en el borde $(42.25 \%)$ y menor en el pastizal $(19 \%)$ $(H=7.21, n=40, g l=2, p<0.05)$ (Figura $2 c)$. 
Para la tasa de remoción, el tratamiento ET (Figura 3a) mostró el menor porcentaje de pérdida de semillas en el tiempo en el interior (menor al 5\%), y el mayor valor en el pastizal ( $\mathrm{G}=1.06, \mathrm{n}=50, \mathrm{gl}=2$, $\chi^{2}=p>0.05$ ). El tratamiento EP (Figura 3b), reveló un significativo incremento en la tasa de remoción de semillas en el interior del bosque $\left(\mathrm{G}=12.19, \mathrm{n}=50, \mathrm{gl}=2, \chi^{2}=\mathrm{p}<0.05\right)$. Por último con el tratamiento SE (Figura 3c) se encontró la mayor tasa de remoción respecto a los demás tratamientos, con un mayor porcentaje de pérdida en el tiempo

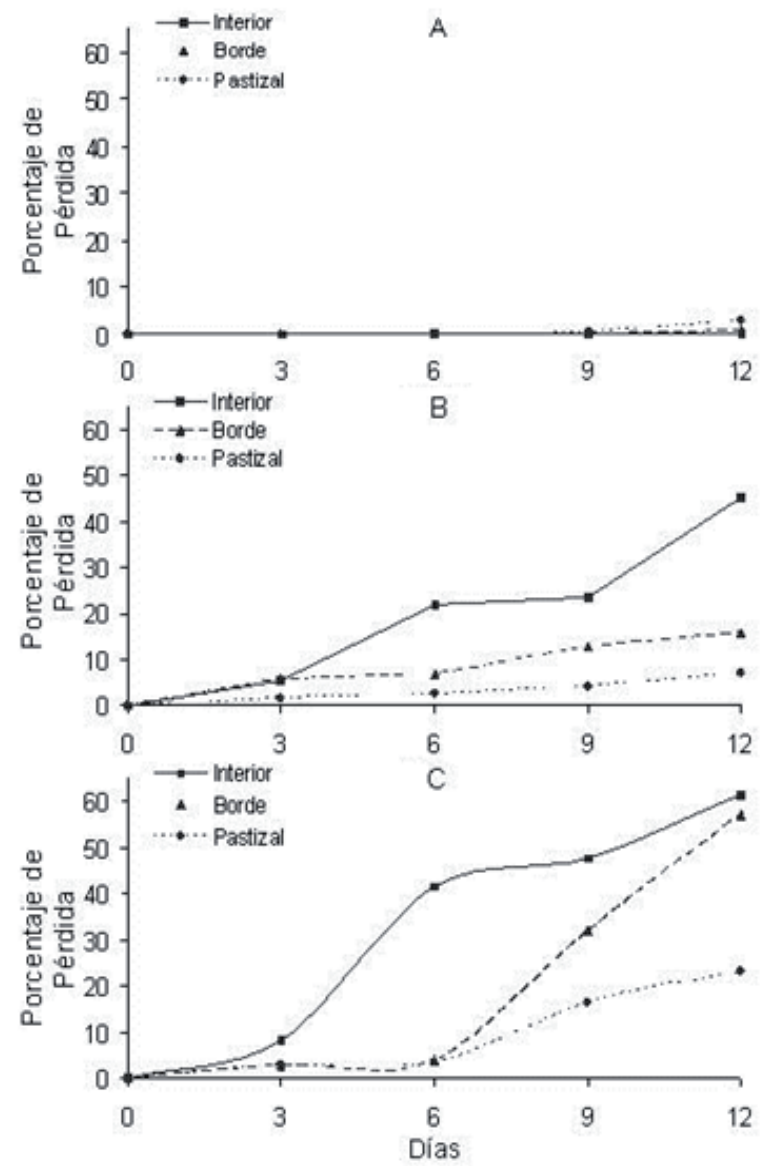

Figura 3. Tasa de remoción de semillas para cada tratamiento en cada zona del experimento 1 (junio). A) Exclusión Total. B) Exclusión Parcial. C) Sin Exclusión.

al interior del bosque ( $>60 \%$ ) y menor en el pastizal ( $<30 \%)(\mathrm{G}=29.91, \mathrm{n}=50, \mathrm{gl}=2$, $\left.\chi^{2}=p<0.05\right)$.

En julio se removieron 1620 semillas luego de 12 días de iniciado el experimento. Durante este tiempo fueron removidas más del $50 \%$
(983) de las semillas de los tratamientos con acceso a roedores al interior del bosque. Al comparar la remoción por tratamientos entre hábitats se encontraron diferencias significativas. La remoción fue mayor para el tratamiento EP en el interior $(\mathrm{H}=33.35$, $\mathrm{n}=50, \mathrm{gl}=2, \mathrm{p}<0.05$ ) (Figura $4 a$ ) y en el borde $(H=9.16, n=26, g l=2, p<0.05)$
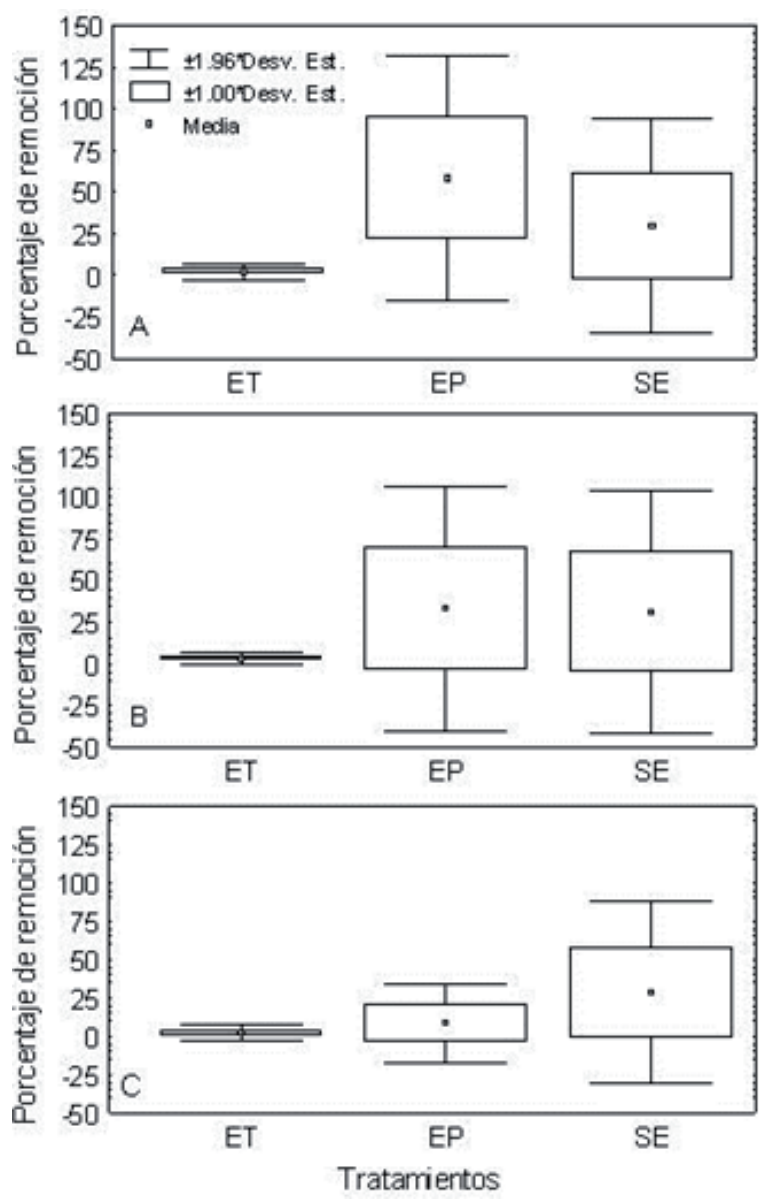

Figura 4. Comparación entre el porcentaje de remoción de semillas entre tratamientos del experimento 2 (julio). A)Interior. B)Borde. C) Pastizal. ET=Exclusión total; $\mathrm{EP}=$ Exclusión parcial; $\mathrm{SE}=\mathrm{Sin}$ exclusión.

(Figura 4b). Por su parte en el tratamiento SE presentó la mayor remoción en el pastizal (pastizal $\mathrm{H}=21.89, \mathrm{n}=48, \mathrm{gl}=2, \mathrm{p}<0.05$ ) (Figura 4c).

Respecto a la ubicación, el tratamiento ET (Figura 5a) presentó la mayor remoción de semillas al interior del bosque $(17 \%)$ y la menor en el borde (14\%); aunque estas 
diferencias no fueron significativas $(\mathrm{H}=2.42$, $n=49, g l=2, p>0.05)$. Por el contrario en el tratamiento EP (Figura 5b) se presentó un porcentaje de remoción significativamente mayor $(\mathrm{H}=20.73, \mathrm{n}=45, \mathrm{gl}=2, \mathrm{p}<0.05)$ en el interior (58.37) del fragmento respecto al pastizal (5.87\%). Finalmente en el tratamiento sin exclusión (SE) (Figura 5c) se presentó un mayor porcentaje de
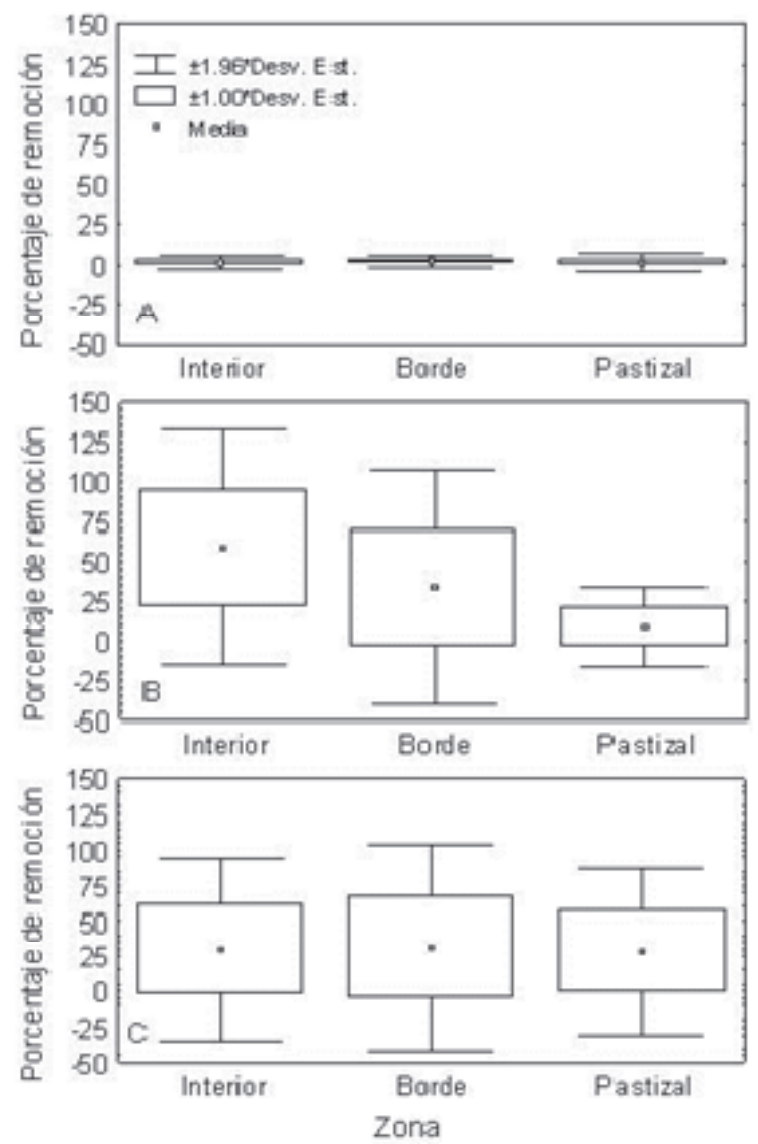

Figura 5. Comparación entre el porcentaje de remoción y la zona trabajada en el fragmento de bosque para cada tratamiento del experimento 2 (julio). A). Exclusión Total. B). Exclusión Parcial. C). Sin Exclusión.

remoción en el borde (24.75\%) respecto al pastizal, aunque estas diferencias no fueron significativas $(H=0.73, n=30, g l=2$ $p>0.05)$.

La tasa de remoción de semillas (Figura 6) fue mayor al interior del fragmento para los tratamientos EP y SE mostrando diferencias significativas $(\mathrm{G}=25.183 .74$, $\mathrm{n}=498, \mathrm{gl}=23, \chi^{2}=\mathrm{p}<0.05, y \mathrm{G}=8.28$,

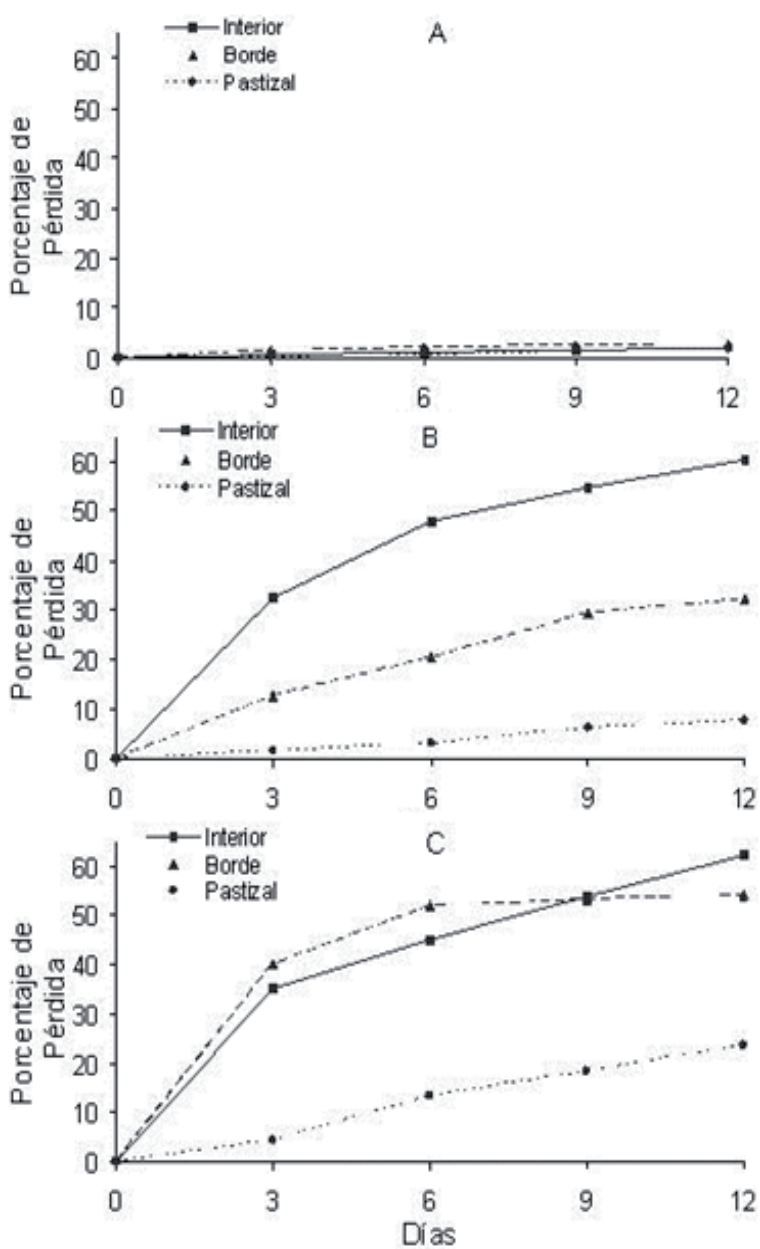

Figura 6. Tasa de remoción de semillas para cada tratamiento del experimento 2 (julio). A) Exclusión Total, B) Exclusión Parcial, C) Sin Exclusión.

$\mathrm{n}=49, \mathrm{gl}=2, \chi^{2}=\mathrm{p}<0.05$ respectivamente). Sin embargo para el tratamiento EP la tasa fue mayor en el pastizal pero sin diferencias significativas $(\mathrm{G}=0.9633, \mathrm{n}=498, \mathrm{gl}=23$, $\left.\chi^{2}=p>0.05\right)$.

\section{DISCUSIÓN}

Captura de roedores. La baja abundancia y variedad de roedores encontrada evidencia el efecto negativo de la fragmentación sobre el ensamblaje de roedores en esta zona. En el caso particular del área de muestreo se le suma el efecto del uso en los pastizales de plaguicidas (glifosato) para el sostenimiento de los pastos mejorados.

Se ha sugerido que la fragmentación influye sobre el desarrollo de las comunidades de 
roedores $(3,4,14,22)$. La intensidad de los efectos de la fragmentación sobre las comunidades animales, varía de acuerdo a la estructura de la vegetación y a condiciones abióticas del fragmento de bosque (17). Becerra-Ramírez (18), sugirió que la composición y abundancia de especies de pequeños mamíferos en el bosque seco varía respecto al grado de alteración o influencia antrópica y a las habilidades que cada especie tenga para adaptarse al nuevo ecosistema.

Respecto al efecto de los plaguicidas sobre los roedores, Vander Wall (23) demostró su efecto sobre las habilidades olfativas de los roedores; encontraron que las moléculas de los plaguicidas se adhieren al suelo, activándose con el aumento de la humedad relativa e incrementando su volatilidad con el aumento de la temperatura. Cuando esta acumulación ocurre cerca al sitio donde se encuentra el alimento de los roedores, altera la facilidad con la que dicho alimento es encontrado, afectando especialmente a especies granívoras, como Heteromys australis, que depositan semillas en escondites $(15,24)$. Esto permite pensar en los plaguicidas como un factor que influye sobre la abundancia de roedores en el área de estudio, especialmente en el pastizal donde su uso es constante y en los bordes, donde puede existir una acumulación (25).

Remoción de semillas. La cuantificación del porcentaje de remoción neta permitió una mejor aproximación a los organismos que atacaron las semillas en cada tratamiento. En ET la remoción estuvo dada por insectos, en el tratamiento EP estuvo dada por roedores y en el tratamiento SE posiblemente estuvo dada por aves y mamíferos mediano.

La remoción por insectos (ET), dentro de los que se observaron hormigas y coleópteros, fue baja durante los dos experimentos. Este resultado concuerda con lo encontrado por Donoso et al (7) quienes analizaron el efecto de la fragmentación sobre la depredación de semillas en el bosque Maulino en Chile; observaron que la granivoría por insectos fue dos veces menor en encierros impermeables ( $\sin$ acceso a vertebrados) que en encierros semi-impermeables (acceso a pequeños vertebrados) y el tratamiento control (sin encierro). Estos autores consideraron que hormigas y coleópteros fueron los principales predadores de semillas dentro del encierro impermeable y que su actividad estuvo afectada por la fragmentación del bosque.

Dentro de los insectos, los coleópteros son considerados grandes predadores de semillas (10), estos generalmente atacan las semillas antes de ser dispersadas, siendo el caso para las semillas de Samanea saman, en donde los bruchidos (Coleoptera) son sus principales depredadores predispersión (26).

Para el tratamiento EP los resultados concuerdan con diversos estudios en los que los roedores han sido considerados como los principales predadores de semillas en diferentes ecosistemas $(1,4,6,8,12)$; siendo afectados por la fragmentación del hábitat $(7,11,27)$.

Respecto al tratamiento SE, los datos sugieren que la posible actividad predatoria por parte de mamíferos medianos y aves es mayor en el borde y en el pastizal. El único mamífero mediano registrado en la zona fue Dasyprocta punctata, el cual aunque es importante depredador de semillas (10), prefiere frutos carnoso con semillas grandes. En este sentido es más probable que la depredación de semillas encontrada en el tratamiento SE haya sido por aves granívoras de los géneros Sporophila, Sicalis y Volantina.

Los resultados de la remoción de semillas por roedores muestran un efecto evidente de la ubicación (interior-borde-pastizal) sobre la pérdida de semillas, siendo mayor en el interior, seguida del borde y del pastizal respectivamente. De acuerdo con Jones et al (12), la variación en los patrones espaciales de la depredación de semillas depende de las densidades poblacionales del depredador. La ausencia de roedores en el pastizal y en los bordes se puede explicar por la baja oferta de alimento, así como a la dificultad en encontrar semillas debido al uso de plaguicidas; adicionalmente los roedores pueden estar evitando las áreas abiertas para minimizar la presión de los 
depredadores. Por su parte, en los bordes existe una presión de depredación alta y una acumulación de contaminantes, en este caso plaguicidas $(23,25)$. La presencia de un roedor que acumula semillas en alta proporción como Heteromys australis se relaciona con el mayor consumo de semillas al interior del bosque. Esta especie puede estar encontrando al interior del bosque unas condiciones para establecerse, menos variables en la humedad del suelo y el aire, una menor exposición a depredadores y una menor concentración de plaguicidas $(8,12,15,16,23)$.

La disponibilidad de alimento durante el primer experimento fue mayor dada la presencia de frutos dentro del bosque, mientras que durante el segundo experimento la disponibilidad de frutos en el bosque fue menor (observación personal). Esta diferencia podría explicar el mayor aprovechamiento de las semillas por los roedores durante el segundo experimento, de tal manera que la densidad de semillas en la zona afecta la remoción en un sitio determinado, sugiriendo que la depredación de semillas en esta zona es un proceso denso-dependiente.

De acuerdo con la hipótesis de escape $(12,28)$, los depredadores de semillas forrajean más en lugares con mayor densidad y variedad de semillas, que es generalmente al interior de los bosques cerca a los parentales; al mismo tiempo, minimizan los costos de búsqueda y el riesgo a la depredación. En consecuencia los roedores pueden evitar lugares donde la densidad de semillas y/o la probabilidad de arribo de estas es menor, que en el caso de este trabajo son los pastizales. Esto puede explicar, la baja remoción de semillas y la baja densidad de los roedores en los pastizales en comparación con el interior del fragmento.

Al haber una menor disponibilidad de frutos y semillas durante la época de la realización del segundo experimento, los puntos donde se colocaron los tratamientos durante este experimento podrían estar simulando el lugar de concentración de semillas cerca a un árbol parental, haciéndola más vulnerable a la acción depredadora de los roedores. Esto podría explicar por qué la tasa de remoción fue mayor durante los primeros días del experimento y se estabilizó luego de un tiempo.

En general, la tasa de remoción de semillas por roedores reflejó su relación con la presencia de estos en los diferentes hábitats del bosque y por consiguiente con su actividad predadora de semillas. Esta actividad pudo estar determinada por cambios en las condiciones bióticas y abióticas entre el interior, el borde y el pastizal a causa de la fragmentación $(7,11,12)$ y por el uso de plaguicidas $(23)$.

En conclusión, los resultados obtenidos en este trabajo indican que los roedores son los principales removedores de semillas en el sector Cerritos-La Virginia, mostrando el mayor porcentaje y la tasa más alta de remoción al interior del fragmento. Se evidencia una relación entre el porcentaje y la tasa de remoción de semillas con la ubicación en el fragmento (interior- bordepastizal). Se reconoce a Heteromys australis como un posible predador de semillas al interior del fragmento.

Aunque es posible que las aves jueguen un papel importante en la remoción de semillas, este trabajo solo permite hacer una aproximación preliminar, por lo que es importante evaluar de manera directa el papel de las aves granívoras en las tasas de remoción de semillas dispersadas en estos fragmentos. Por último sugerimos evaluar el papel de los roedores en la tasa de depredación de plántulas, ya que aunque los propágulos escapen a la depredación en el estadío de semilla, siguen siendo vulnerables a la acción depredadora de los roedores en estados subsiguientes. Asi se tendría una idea más completa del papel que los depredadores de semillas y plántulas tienen sobre los procesos de sucesión y regeneración natural, especialmente en áreas degradadas. 


\section{Agradecimientos}

Gustavo Pueyo, John Mario Rodríguez M.Sc, Germán Jiménez M.Sc, Amanda Varela Ph.D., Michael Alberico (q.e.p.d.), Catalina Sánchez. Gabriel Vélez y Elsa de Vélez. Este trabajo formó parte del proyecto "Composición de la comunidad de mamíferos no voladores en fragmentos de bosque en la Loma del Oso (área Cerritos de la Virginia, Municipio de Pereira, Depto. Risaralda).", financiado por la Pontificia Universidad Javeriana (ID 000634).

\section{REFERENCIAS}

1. Paine CET, Beck H. Seed predation by neotropical rainforest mammals increases diversity and seedling recruitment. Ecology 2007; 88(12):3076-3087.

2. Forget PM, Wenny D. How elucidate seed fate? A review of methods used to study seed removal and secondary seed dispersal. En: Forget PM, Lambert JE, Hulme PE and Vander Wall SB. Seed fate: predation, dispersal and seedling stablishment. United Kingdom: CABI Publishing; 2004.

3. Cole R. Postdispersal seed fate of tropical montane trees in an agricultural landscape, southern Costa Rica. Biotropica 2009; 41(3): 319-327.

4. Hoshizaki K, Miguchi $H$. Influence of forest composition on tree seed predatrion and rodent response: a comparison of monodominant and mixed temperate forest in Japan. En: Forget PM, Lambert JE, Hulme PE and Vander Wall SB. Seed fate: predation, dispersal and seedling stablishment. United Kingdom: CABI Publishing; 2004.

5. DeMattia EA, Curran LM, Rathcke BJ. Effects of small rodents and large mammals on neotropical seeds. Ecology 2004; 85(8):2161-2170.

6. Schnurr JL, Canham CD, Ostfeld RS, Inouye RS. Neighborhood analyses of small-mammal dynamics: Impacts on seed predation and seedling establishment. Ecology 2004; 85(3):741-755.
7. Donoso DS, Grez AA, Simonetti JA. Effects of forest fragmentation on the granivory of differently sized seeds. Biol Cons 2003; 115:63-70.

8. Fedriani JM, Manzaneda AJ. Pre and postdispersal seed predation by rodents: balance of food and safety. Behav Ecol 2005; 8:1018-1024.

9. LoGiudice K, Ostfeld RS. Interactions between mammals and trees: predation on mammal-dispersed seeds and the effect of ambient food. Oecol 2002; $130: 420-425$.

10. Notman EM, Villegas AC. Patterns of seed predation by vertebrates vs. invertebrate seed predation among different plants species, season and spatial distribution. En: Forget PM, Lambert JE, Hulme PE and Vander Wall SB. Seed fate: predation, dispersal and seedling stablishment. United Kingdom: CABI Publishing; 2004.

11. Holl KD, Lulow ME. Effects of species, habitat, and distance from the edge on post- dispersal seed predation in a tropical rainforest. Biotropica 1997; 29(4):459-468.

12. Jones FA, Peterson CJ, Haines BL. Seed predation in neotropical premontane pastures: site, distance, and species effects. Biotropica 2003; 35(2):219-225. 
13. García-Orth X, Martínez-Ramos M. Seed Dynamics of Early and Late Successional Tree Species in Tropical Abandoned Pastures: Seed Burial as a Way of Evading Predation. Restoration Ecol 2008; 16(3):435-443.

14. Püttker $T$, Pardini $R$, Meyer-Lucht $Y$, Sommer S. Responses of five small mammal species to micro-scale variations in vegetation structure in secondary Atlantic Forest remnants, Brazil. BMC Ecology 2008; 8:9.

15. Lambert TD, Malcolm JR, Zimmerman BL. Amazonian small mammal abundances in relation to habitat structure and resource abundance. J Mammal 2006; 87(4):766-776.

16. Christianini AV, Galetti M. Spatial variation in post-dispersal seed removal in an Atlantic forest: Effects of habitat, location and guilds of seed predators. Acta Oecológica 2007; 32(3):328-336.

17. Fahrig L. Effects of habitat fragmentation on biodiversity. Annu Rev Ecol Syst 2003; 34:487-515.

18. Becerra-Ramírez MT. Influencia del disturbio antrópico sobre las comunidades de pequeños mamíferos de bosque seco tropical. [Tésis de Maestría]. Bogotá D.C.: Universidad Nacional de Colombia, Facultad de Ciencias; 2002.

19. Chauvet S, Forget PM. Edge effects on post-dispersal seed removal in a ragmented rain forest in French Guiana. J Trop Ecol 2005; 21:113-116.

20. Ministerio del Medio Ambiente. Agenda ambiental del Municipio de Pereira. Bogotá D.C.: Ministerio del Medio Ambiente; 2002.
21. Durr PA. The biology, ecology and agroforestry potential of the raintree, Samanea saman (Jacq.) Merr. Agroforestry systems 2001; 51:223-237.

22. Pardini R. Effects of forest fragmentation on small mammals in an Atlantic Forest landscape. Biodiversity and Conservation 2004; 13:2567-2586.

23. Vander Wall SB. How rodents smell buried seeds: a model based on the behavior of pesticides in soil. J Mammal 2003; 84(3): 1089-1099.

24. Orland MC, Kelt AD. Responses of a Heteromyid rodent community to largeand-small-range dynamics. J Mammal 2007; 88(5):1280-1287.

25. Weathers KC, Cadenasso ML, Pickett STA. Forest edges as nutrient and pollutant concentrators: potential synergisms between fragmentation, forest canopies, and the atmosphere. Conserv Biol 2001; 15(6):1506-1514.

26. Cascante A, Quesada M, Lobo JJ, Fuchs EA. Effects of dry tropical forest fragmentation on the reproductive success and genetic structure of the tree Samanea saman. Conserv Biol 2002; 16(1):137-147.

27. Fleury M, Galetti M. Forest fragment size and microhabitat effects on palm seed predation. Biol Cons 2006; 131:1-13.

28. Janzen $\mathrm{DH}$. Seed predation by animals. Annu Rev Ecol Syst 1971; 2:465-492. 